\title{
Why cars in the next lane seem to go faster
}

\author{
The temptation to change lanes on a motorway may be prompted by an illusion.
}

S witching lanes while driving along a busy road can be a risky manoeuvre. It is often instigated on the driver's judgement that the cars in the next lane are moving faster than those in the driver's own lane. But faulty intuition ${ }^{1-3}$ may cause people to overestimate the speed of vehicles in the next lane, believing that they are moving faster even when both lanes have the same average speed. We suggest that this illusion occurs because more time is generally spent being overtaken (passed) by other vehicles than is spent in overtaking them. Knowing that this effect is illusory might encourage drivers to resist small temptations to change lanes.

We used computer simulations to create two lanes of traffic with identical characteristics, except that their congestion varied depending on random starting gaps. Vehicles then accelerated if no other vehicle was within the minimum headway distance, otherwise they decelerated. Minimum headway distances increased with higher velocities to prevent collisions.

We evaluated statistically the movements of an individual driver compared with all the others in the next lane. Under baseline conditions, many one-second epochs produced a change in the driver's relative position. Epochs in which the index vehicle was overtaken were more frequent than epochs in which the index vehicle was overtaking another vehicle (Fig. 1). The total number of vehicles that passed the driver was balanced by the total number of vehicles that were overtaken by the driver because of multiplicity in some epochs.

Doubling the acceleration generated a larger difference, and halving the minimum headway distance, to represent 'tailgating,' greatly increased the difference. Reducing the frequency with which the index driver glanced at the next lane from once every second to once every two seconds attenuated the difference. No combination yielded a slower apparent speed for the next lane.

We also videotaped traffic sequences by mounting a camera in a moving vehicle and filming the side-view perspective of the next lane on a congested road. When a section of videotape showing a slightly slower average speed in the next lane was screened to driving students $(n=120), 70 \%$ stated that the next lane was moving faster and $65 \%$ said they would change lane if possible.

From these results, we suggest that drivers are responding to an illusion: namely, that the next lane on a congested road appears to be moving faster than the driver's present lane, even when both lanes have the same

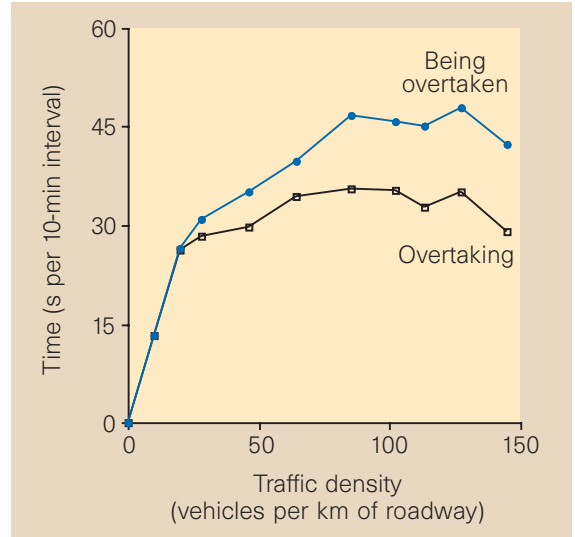

Figure 1 Relation of traffic density to the amount of time spent overtaking and being overtaken. All time estimates have standard errors of less than 5 seconds that decrease with traffic density. For example, a driver travelling for 10 minutes on a roadway with congestion of about 100 vehicles per kilometre would spend 47 seconds being overtaken by vehicles in the other lane and about 35 seconds overtaking vehicles in the other lane if both lanes have the same average speed. Details of simulation models are available from the authors.

average speed. This occurs because vehicles spread out when moving quickly and pack together when moving slowly. A driver can therefore overtake many vehicles in a brief time interval, but it takes much longer for the driver to be overtaken by the same vehicles.

Other aspects of human perception may accentuate the impression that the next lane is moving faster. Differential surveillance can occur because drivers look forwards rather than backwards, so vehicles that are overtaken become invisible very quickly, whereas vehicles that overtake the index driver remain conspicuous for much

\section{Rare-earth metals}

\section{Is gadolinium really ferromagnetic?}

Gadolinium is accepted to be one of the four ferromagnetic elements, along with iron, cobalt and nickel, although its Curie point, $T_{\mathrm{C}}$ (the temperature above which ferromagnetism is lost), is only $292 \mathrm{~K}$. Ferromagnets exhibit a characteristic divergence of their susceptibility at $T_{C}$, but no such divergence in any direction is found for needle-shaped crystals of gadolinium. Instead, the susceptibility diverges at a lower spin-reorientation temperature, $T_{\text {sr }}$, of 225 $\mathrm{K}$, where the anisotropy changes sign. We propose that the magnetic order between longer ${ }^{4}$. Moreover, a driver is more likely to glance at the next lane for comparison when he is relatively idle ${ }^{5}$ while moving slowly.

Even if attention was not focused in particular directions and was evenly spaced in time, human psychology may make being overtaken (losing) seem more salient than the corresponding gains ${ }^{6}$. Furthermore, misconceptions about randomness can make runs of overtaking and being overtaken seem unduly prolonged ${ }^{7,8}$. Our study highlights the effects of congestion and the increasing importance of the illusion, given that the number of miles travelled by vehicles is increasing at a much faster rate than the amount of roadway ${ }^{9,10}$.

Donald A. Redelmeier*,

Robert J. Tibshirani $\uparrow$

*University of Toronto, Sunnybrook and Women's College Health Sciences Centre,

G-151, 2075 Bayview Avenue,

Toronto, Ontario M4N 3M5, Canada

e-mail:dar@ices.on.ca

$\dagger$ Departments of Health Research and Policy and of Statistics, Stanford University,

Stanford, California 94305-5405, USA

1. Snowden, R. J., Stimpson, N. \& Ruddle, R. A. Nature 392, 450 (1998).

2. Walton, D. \& Bathurst, J. Accident Anal. Prevent. 30, 821-830 (1998).

3. Tversky, A. \& Kahneman, D. Q. J. Econ. 107, 1039-1061 (1991) 4. Larson R. C. Operat. Res. 35, 895-904 (1987).

5. Angrilli, A., Cherubini, P., Pavese, A. \& Manfredini, S. Percept. Psychophys. 59, 972-982 (1997).

6. Tversky, A. \& Kahneman, D. Science 185, 1124-1131 (1974).

7. Gilovich, T., Vallone, B. \& Tversky, A. Cogn. Psychol. 17, 295-314 (1985)

8. Feller, W. An Introduction to Probability Theory and its Applications Vol II (Wiley, New York, 1966).

9. Helbing, D. \& Huberman, B. A. Nature 396, 738-740 (1998). 10. Bureau of the Census Statistical Abstract of the United States 1996 116th edn (Government Printing Office, Washington DC, 1997).

$T_{\text {sr }}$ and $T_{\mathrm{C}}$ is not truly ferromagnetic, but is akin to the incommensurate order found in erbium.

It used to be thought ${ }^{1}$ that gadolinium had a helical spin structure similar to that of terbium, dysprosium and holmium, but that it became ferromagnetic when a small field $\left(\sim 1 \mathrm{kA} \mathrm{m}^{-1}\right)$ was applied. This idea was discounted after neutron diffraction ${ }^{2}$ showed that the turn angle must be smaller than 2 degrees. Gadolinium is considered to be the only simple ferromagnet among the rare-earth metals. Moments lie along the hexagonal $c$-axis between $T_{\mathrm{C}}$ and $T_{\mathrm{sr}}$, where they become inclined at an angle to $c$ which reaches 65 degrees at $180 \mathrm{~K}$.

Previous studies ${ }^{3}$ of the anisotropic susceptibility, $\chi=M / H$, where $M$ is the 
magnetization and $H$ is the magnetic field strength, have been performed on crystals with a demagnetizing factor, $N$, of 0.1 to 0.3 . The demagnetizing factor depends only on shape and determines the strength of the reverse field that appears inside a sample when it is magnetized; $\chi$ is limited by the demagnetizing effect to $1 / N$. We therefore use long, thin samples with $N \approx 0.01$, and measure $\chi$ in a small alternating-current field of $8 \mathrm{~A} \mathrm{~m}^{-1}$, compensating for the Earth's field with a pair of Helmholz coils.

Figure 1a shows that the real component $\chi^{\prime}(T)$ measured perpendicular to the $c$-axis has a sharp peak at the magnetic transition with a maximum value of $49 \pm 4$. As the temperature decreases further, $\chi^{\prime}$ increases to saturate near the demagnetizing-limited value $1 / N=77$. When the field is parallel to $c$, there is only a shoulder in $\chi^{\prime}(T)$ at $T_{\mathrm{C}}$ and the value does not exceed 10. $\chi^{\prime}(T)$ increases at lower temperature, reaching the demagnetizing limit $1 / N=66$ at $230 \mathrm{~K}$.

Figure $1 \mathrm{~b}$ shows the real and imaginary components, $\chi^{\prime}$ and $\chi^{\prime \prime}$, for the two directions in the vicinity of $T_{\mathrm{C}}$, after correcting for the demagnetizing effect ${ }^{4}$. The $\chi_{\perp}{ }^{\prime}(T)$ peak at the magnetic transition is accompanied by a small peak in the $\chi_{\perp}{ }^{\prime \prime}(T)$ component, but $\chi_{\|}{ }^{\prime}(T)$ barely exceeds 10 at $T_{\mathrm{C}}$ and $\chi_{\|}^{\prime \prime}(T)$ is zero within experimental error. There is no evident divergence at $T_{\mathrm{C}}$. To see whether the observed non-divergence of the $c$-axis susceptibility could be related to pinning of domain walls, we applied a $50-\mathrm{Hz}$ alternating-current bias field of $80 \mathrm{~A} \mathrm{~m}^{-1}$ to mobilize the walls. The form of $\chi^{\prime}$ and $\chi^{\prime \prime}$ did not change.

The susceptibility of a ferromagnet normally diverges as $|\epsilon|^{-\gamma}$, where $\epsilon=\left(T-T_{\mathrm{C}}\right) / T_{\mathrm{C}}$ and the critical exponent $\gamma$ is 1.38 for isotropic spins (Heisenberg model) or 1.24 for extreme uniaxial anisotropy (Ising model). The presence of uniaxial dipolar anisotropy ${ }^{5}$ should not prevent divergence at $T_{\mathrm{C}}$.

Internal checks on the anomalous behaviour of $\chi_{\|}{ }^{\prime}(T)$ are provided by the divergence at lower temperatures in both directions and by the peak in $\chi_{\perp}(T)$. The susceptibility effectively reaches the demagnetizing-limited values at the spinreorientation transition, but not at the Curie point. The sharp peak observed at $T_{\mathrm{C}}$ when the field is applied in the basal plane resembles the peak at the Néel point (at which anti-ferromagnetic materials become paramagnetic), $T_{\mathrm{N}}$, of $230 \mathrm{~K}$ for terbium, where an in-plane helical structure is established ${ }^{6}$ and $\chi^{\prime}$ reaches a value of 8 . When magnetized in-plane near $T_{\mathrm{C}}$, where magnetocrystalline anisotropy is negligible, the susceptibility of gadolinium resembles that of a long-period helix rather than a true ferromagnet. When magnetized along $c$,

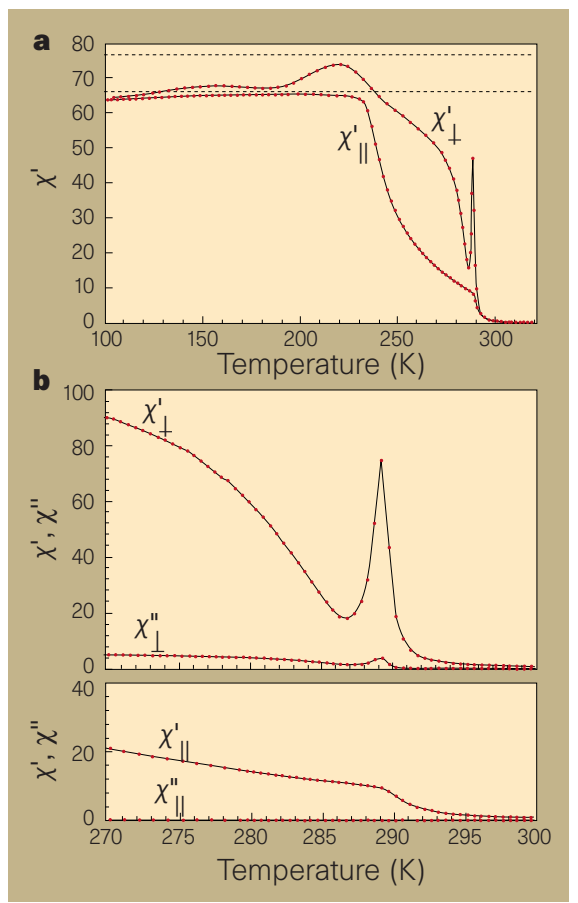

Figure 1 Magnetic susceptibility of a gadolinium crystal. a, Real and imaginary parts of the susceptibility measured with an alternating-current technique on long samples cut from a crystal of gadolinium with the field applied parallel (||) and perpendicular $(\perp)$ to the $c$-axis. The susceptibility is independent of frequency in the range $10 \mathrm{~Hz}$ to $1 \mathrm{kHz}$. The horizontal dotted lines indicate the saturation values expected for a divergent susceptibility, given the sample shape. b, Internal susceptibility of gadolinium in the vicinity of $T_{c}$, corrected for the demagnetizing effect caused by the sample shape.

there is a long-period sinusoidally modulated structure like that of erbium.

The value of the exchange stabilization energy of the helix, $J\left(q_{\mathrm{m}}\right)-J(0)$, may be estimated from the peak susceptibility using $\chi_{\max }=\mu_{0} N m^{2} /\left\{S^{2}\left[J\left(q_{\mathrm{m}}\right)-J(0)\right]\right\} \quad($ ref. 7), where $\mu_{0}$ is the permeability of free space, $m$ is the atomic moment of gadolinium, $S=7 / 2, J$ is the exchange energy and $q_{\mathrm{m}}$ is the reduced wave vector in the $c$-direction; $\chi_{\max }=75$ gives $J\left(q_{\mathrm{m}}\right)-J(0)=0.013 \mathrm{~K}$. Extrapolating from the heavy rare-earth metals, where $J\left(q_{\mathrm{m}}\right)-J(0) \approx \mathrm{q}_{\mathrm{m}}{ }^{4}$, we estimate that $q_{\mathrm{m}}=0.029 \AA^{-1}$; the corresponding wavelength, $\lambda_{\mathrm{m}}$, is $70 \AA$.

Examination of gadolinium close to $T_{\mathrm{C}}$, using techniques to observe the long-period modulation directly, may confirm that gadolinium is not really ferromagnetic.

J. M. D. Coey, V. Skumryev, K. Gallagher

Physics Department, Trinity College,

Dublin 2, Ireland

1. Belov, K. P. \& Pedko, A. L. Zh. Eksp. Teor. Fiz. 47, 87 (1962). (English transl., Sov. Phys. JETP 15, 62-64 (1962).

2. Cable, J. W. \& Wollan, E. O. Phys. Rev. 165, 733-734 (1968).

3. Dan'kov, S. Y., Tishin, A. M., Pecharsky, V. K. \& Gschneider,

K. A. Jr Phys. Rev. B 57, 3478-3489 (1998).

4. Chen, D.-X. \& Sanchez, A. J. Appl. Phys. 70, 5463-5477 (1991).

5. Geldart, D. J. W., Hargraves, P., Fujiki, N. M. \& Dunlap, R. A. Phys. Rev. Lett. 62, 2728-2731 (1989).

6. del Moral, A. \& Lee, E. W. J. Phys. F 4, 280-290 (1974).

7. Kitano, Y. \& Nagamia, T. Prog. Theor. Phys. 31, 1-37 (1964)

\section{Physiology}

\section{Warm feet promote the rapid onset of sleep}

Even healthy people occasionally have difficulty falling asleep. Psychological relaxation techniques, hot baths, soothing infusions of plant extracts, melatonin and conventional hypnotics are all invoked in the search for a good night's sleep. Here we show that the degree of dilation of blood vessels in the skin of the hands and feet, which increases heat loss at these extremities, is the best physiological predictor for the rapid onset of sleep. Our findings provide further insight into the thermoregulatory cascade of events that precede the initiation of sleep ${ }^{1}$.

Our analysis combines data from two intervention studies designed to induce phase shifts in the circadian pacemaker ${ }^{2,3}$. Healthy young men were given melatonin, bright light, or both, in the evening $(n=8)$, or a large carbohydrate-rich meal in either the morning or the evening $(n=10)$. These manipulations had different thermoregulatory effects and so gave a broad variance, enabling us to extract the best predictor variables for the latency of sleep onset.

Subjects lay in bed under controlled conditions (lighting less than 8 lux; $22^{\circ} \mathrm{C}$ ), taking small snacks of constant caloric value every hour during wakefulness. The latency of sleep onset was defined as the time between lights out (24:00) and the first occurrence of stage 2 in the sleep EEG recordings. Heart rate, core body temperature (rectal) and proximal (combined infraclavicular, thigh, stomach, forehead) and distal (combined hands and feet) skin temperatures were continuously measured ${ }^{4}$ (and later collapsed into 30-min bins). Sleepiness was rated ${ }^{2}$ every $30 \mathrm{~min}$ and saliva was collected for melatonin assay ${ }^{5}$. We calculated the distal-proximal temperature gradient (DPG), a measure of blood flow in distal skin regions (efficiently regulated by artereovenous anastomoses $)^{6}$ that provides an indirect index of distal heat loss.

By using a multiple regression model for repeated measures, with the latency of sleep onset as the dependent variable $(n=18$ subjects, 144 data points; between-subjects differences taken into account), we found the highest correlation with the DPG averaged over the three data points between $22: 30$ and 24:00 $(r=-0.47, P<0.0001)$. Thus, the greater the distal vasodilation in the late evening, the shorter was the time taken to fall asleep. We found that correlations were weaker with subjective sleepiness ratings $(r=-0.33, P<0.0001)$, core body temperature $(r=0.26, P<0.005)$, melatonin $(r=-0.15$, n.s.; after excluding the melatonin intervention data), rate of change of core body temperature $(r=-0.05$, n.s. $)$ and heart rate $(r=0.05$, n.s. $)$. 